\title{
DOCSCOLLAB : UMA FERRAMENTA DE AUXÍLIO À EDIÇÃO COLABORATIVA
}

\author{
Maria de Fátima W. do Prado Lima - CCTI-UCS - mfwplima@ucs.br \\ Daniel Battistelo - CCTI-UCS - dbattistelo@gmail.com \\ Carine G. Webber - CCTI-UCS - cgwebber@ucs.br \\ Bruno B. Guimarães - CCTI-UCS - bbguimar@ucs.br
}

\begin{abstract}
Resumo. Ferramentas de suporte à edição colaborativa visam contribuir para o desenvolvimento da expressão escrita, habilidade tão necessária em todas as disciplinas e níveis educacionais. Este artigo propõe um estudo sobre editores colaborativos e apresenta uma ferramenta web simples que adiciona novas funcionalidades de coordenação, cooperação $e$ comunicação ao Google Docs. A ferramenta desenvolvida possibilita a criação de um fórum de discussão cujos tópicos estejam vinculados a documentos em processo de edição. Além disso, ela permite a criação de uma árvore de alocação de tarefas que auxilia no gerenciamento das atividades do grupo envolvido na edição colaborativa de documentos. A ferramenta é operacional e está disponível para usuários através de sua instalação em servidor local.
\end{abstract}

Palavras-chave: Escrita Colaborativa, Colaboração, Software educacional.

\section{DOCSCOLLAB: A COLLABORATIVE EDITING TOOL}

\begin{abstract}
Collaborative writing tools intend to contribute to writing skills acquisition as an important ability for students at any discipline and educational level always. This article presents a simple web tool which adds some coordination, cooperation and communication features to Google Docs. Through this tool users can create web forums having messages linked to personal documents. It also allows the creation of a task list tree which helps the task management of members during collaborative edition. The tool is fully operational and can be accessed thorough an installation at a local server.
\end{abstract}

Keywords: Collaborative writing, Collaboration, Educational Software.

\section{Introdução}

Uma atividade colaborativa é aquela onde os participantes trabalham em grupos em um problema ou tarefa compartilhada, para a qual se espera que todos contribuam. Uma vez que os participantes possuem competências e habilidades similares ou complementárias, eles devem estabelecer objetivos compartilhados claros e trabalhar juntos nas tarefas. 
Em cenários de colaboração, as tecnologias assumem papéis importantes na comunicação, mediação e motivação, contribuindo nos processos de interação e aprendizagem. Além disso, atividades colaborativas mediadas pelo computador podem ser realizadas sem que fatores como localização e tempo interfiram no sucesso da tarefa.

A abordagem da aprendizagem colaborativa se fundamenta nas teorias que defendem que a aprendizagem é um processo social, pois as pessoas aprendem melhor com e a partir de outros (Resnick, 1991; Salomon e Perkins, 1998). Neste caso, o professor assume o papel de acompanhar e mediar a aprendizagem, intervindo para apresentar novos problemas, validar ou invalidar hipóteses. Existe uma hierarquia vinculada a esse processo, onde deve haver a definição de como cada colaborador interage com os demais e quais os seus privilégios e atividades (Fish, 1988). Neste processo é importante deixar claro a todos os participantes quais os objetivos que devem ser alcançados, quais seus papéis e suas responsabilidades (Neuwirth, 1994).

Estudos sobre a aprendizagem colaborativa são fundamentados e influenciados pelos trabalhos de Vygotsky (1962, 1978). Autores recentes como Fuks (2001) afirmam que um grupo pode produzir melhores resultados do que um indivíduo sozinho pois diferentes interpretações sobre um assunto vêm à tona enriquecendo o processo. Além disso, um grupo é capaz de identificar inconsistências e falhas onde um indivíduo sozinho levaria mais tempo e talvez não identificaria. Um grupo em geral agrega diferentes visões e perspectivas úteis em processos de tomada de decisão, produzindo mais e melhores ideias e alternativas. Um grupo pode também avaliar as opções existentes de maneira mais rápida e pesquisar novas fontes de informação, o que contribui para uma produção significativa e mais elaborada. Além da aprendizagem esperada, atividades de colaboração contribuem para que soluções originais, conceitos e novas ideais possam emergir.

Em se tratando de tarefas de colaboração, as ferramentas de suporte à edição colaborativa podem contribuir fortemente para o desenvolvimento das habilidades ligadas à expressão escrita, tão necessárias em todos os níveis educacionais. Denominados editores colaborativos, tais ferramentas permitem a produção de um documento de modo colaborativo. Para que a escrita colaborativa seja suportada é essencial que os editores colaborativos possuam mecanismos que possibilitem a comunicação afim de que o desenvolvimento do documento possa evoluir de uma maneira construtiva e concisa (Adkins et al., 1999).

O uso de editores colaborativos deve ser simples a fim de que o usuário, autor do documento, possa se dedicar ao seu objetivo principal (Duin, 1990). Entende-se porém que editores colaborativos de uso educacional devem possuir funcionalidades de coordenação e acompanhamento da execução das tarefas colaborativas. Em nosso estudo foram analisados seis editores colaborativos (Google Docs, Zoho Writer, Gobby, ACE, SyncroEdit e EquiText), considerando critérios de comunicação, colaboração e acompanhamento das tarefas. Os resultados desta análise são apresentados na seção 2 deste artigo. A partir deste estudo prévio, a seção 3 descreve a ferramenta DocsCollab, integrada ao Google Docs a fim de agregar funcionalidades ausentes e necessárias à esta aplicação para uso educacional. Para finalizar, a seção 4 apresenta as considerações finais deste artigo e trabalhos futuros.

\section{Análise de Editores Colaborativos Existentes}

Diversas funcionalidades vêm sendo estudadas e implementadas em editores colaborativos a fim de apoiar atividades educacionais. Recursos que auxiliam na percepção do trabalho do grupo são especialmente interessantes pois permitem que 
todos os seus integrantes visualizem o trabalho que está sendo realizado em tempo real. Tais recursos além de úteis em ambientes de escrita colaborativa síncrona têm sido considerados cruciais para que a efetiva colaboração possa acontecer (Gutwin and Greenberg, 2001). Para oferecer esse recurso, o sistema deve possuir algumas funcionalidades tais como a visão de radar estendida (Tran, Yang e Raikundalia, 2006), permitir a visualização da miniatura do documento, e apresentar onde se encontra o cursor dos colaboradores no documento, destacando a área que está sendo alterada ou visualizada pelos mesmos.

Outro recurso importante é o acompanhamento das alterações no documento compartilhado em tempo real. A maioria das ferramentas oferece um bom suporte quando o documento é alterado de maneira assíncrona, geralmente com um painel que permite visualizar as modificações que foram realizadas, ordenadas conforme a sua localização no documento. Para o modo síncrono de colaboração, os autores Tran, Yang e Raikundalia (2006) propõe um recurso denominado diretor de modificações o qual exibe as modificações realizadas no documento de forma ordenada temporalmente (conforme o dia e a hora em que as modificações ocorreram). $\mathrm{O}$ diretor de modificações pode distinguir entre múltiplas alterações que possam vir a ocorrer em um mesmo local do arquivo a fim de que o responsável pelo texto tome conhecimento das alterações que ocorreram no documento.

Rakandalia e Zhang (2005) citam outros recursos para apoiar a percepção do grupo quanto às atividades desenvolvidas pelos participantes em tempo real. Tais recursos foram identificados através de testes realizados utilizando a ferramenta REDUCE - Real Time Distributed Unconstrained Cooperative Editor (Yang et al. 2000). REDUCE é uma ferramenta que permite que vários usuários possam editar um mesmo texto de maneira síncrona estando geograficamente dispersos sem que haja nenhuma restrição durante a colaboração. Os autores propõe a integração de ferramentas para a alocação de tarefas, lista de ação dos usuários e histórico das alterações dos usuários. O objetivo ferramenta de alocação de tarefas é deixar claro para os colaboradores, quem é responsável por qual parte do documento além de deixar os usuários cientes sobre o que será trabalhado em cada seção do documento. A lista de ação de usuário é um recurso que possibilita aos usuários visualizarem o que está sendo produzido pelos demais colaboradores. Essa lista deve registrar ações do tipo: usuário iniciou inserção de texto, apagou certo conteúdo, está editando no momento, parou a edição, entre outros. O compartilhamento das ações em andamento orienta os usuários quanto às tarefas que ele pode desenvolver ou interromper.

Já o editor iWrite, além das funcionalidades de edição utiliza recursos sofisticados de reconhecimento de linguagem natural e correção automática de texto para fornecer feedback automático aos estudantes. Este editor foi desenvolvido a partir da plataforma Google para tarefas de aprendizagem colaborativa para alunos de cursos de Engenharia (Calvo et.al, 2011). Na aprendizagem de língua estrangeira, autores como Woo et al. (2011) defendem o uso de editores como Wikis para uma aprendizagem colaborativa onde os alunos interagem, corrigem e redigem textos colaborativamente. Experimentos como este têm sido realizados em escolas chinesas obtendo resultados satisfatórios na aprendizagem da língua inglesa.

Weng e Gennari (2004) exploram o recurso de anotações nos documentos colaborativos com o objetivo de oferecer um melhor suporte à escrita colaborativa assíncrona. A proposta de tal abordagem, segundo eles, é oferecer um suporte adequado às anotações com o objetivo de melhorar a coordenação e a percepção do grupo. Para isso propõem um modelo de anotação que possua: uma descrição do objeto, da seção a qual a anotação está atrelada, comentário da anotação, criador da anotação, destinatários 
da anotação, data de criação da anotação, prazo de resposta, respostas geradas, status, categorias de anotações, avaliação e urgência.

Percebe-se que em processos de colaboração, os recursos de coordenação, cooperação e comunicação são importantes para o bom funcionamento do processo. A fim de avaliar como estes recursos estão disponíveis nas ferramentas de escrita colaborativa, selecionou-se 6 editores para teste: Google Docs, Zoho Writer, Gobby, ACE, SyncroEdit e EquiText. A tabela 1 apresenta uma comparação entre os editores segundo os recursos disponibilizados por eles (primeira coluna da tabela).

A primeira ferramenta avaliada foi o Google Docs. Ele possibilita a inserção de documento e comentários no documento, o envio de e-mail para os demais colaboradores, o upload dos documentos e a definição básica de papéis. O editor Zoho Writer oferece ao grupo funcionalidades para que a equipe possa se organizar, trocar ideias e informações para a produção do documento. Os editores Gobby e Ace são similares pois apresentam as mesmas características. O Gobby oferece também a funcionalidade de chat.

Tabela 1. Análise Final dos Editores

\begin{tabular}{|c|c|c|c|c|c|c|}
\hline Requisitos & $\begin{array}{c}\text { Google } \\
\text { Docs }\end{array}$ & $\begin{array}{c}\text { Zoho } \\
\text { Writer }\end{array}$ & Gobby & $\begin{array}{l}\text { Synchro } \\
\text { Edit }\end{array}$ & ACE & EquiText \\
\hline Agenda & $x$ & $x$ & $x$ & $x$ & $x$ & $x$ \\
\hline Anotações/Comentários & $\sqrt{ }$ & $\checkmark$ & $x$ & $x$ & $x$ & $\checkmark$ \\
\hline Atribuição de Tarefas & $x$ & $\checkmark$ & $x$ & $x$ & $x$ & $x$ \\
\hline Chat & $x$ & $\checkmark \sqrt{ }$ & $\checkmark$ & $\checkmark$ & $x$ & $x$ \\
\hline Controle de Versão & $\checkmark$ & $\checkmark$ & $x$ & $x$ & $x$ & $x$ \\
\hline E-mail & $\sqrt{ }$ & $x$ & $x$ & $x$ & $x$ & $x$ \\
\hline Formatação de Texto & $\checkmark$ & $\sqrt{ }$ & $x$ & $\sqrt{ }$ & $x$ & $x$ \\
\hline Fórum & $x$ & $x$ & $x$ & $x$ & $x$ & $x$ \\
\hline Importação/Exportação de arquivos texto & $\checkmark$ & $\checkmark$ & $x$ & $x$ & $x$ & $x$ \\
\hline Notificações & $x$ & $x$ & $x$ & $x$ & $x$ & $x$ \\
\hline Papéis & $\sqrt{ }$ & $\sqrt{ } \sqrt{ }$ & $x$ & $x$ & $x$ & $x$ \\
\hline $\begin{array}{l}\text { Paradigma WYSIWIMS (What You See Is } \\
\text { What I May See) }\end{array}$ & $x$ & $x$ & $x$ & $x$ & $x$ & $\checkmark$ \\
\hline $\begin{array}{l}\text { Paradigma WYSIWIS (What You See Is } \\
\text { What I See) }\end{array}$ & $\checkmark$ & $\checkmark$ & $\checkmark$ & $\checkmark$ & $\checkmark$ & $\checkmark$ \\
\hline Percepção de Grupo & $\checkmark$ & $\sqrt{ } \sqrt{2}$ & $\sqrt{ } \sqrt{ }$ & $\checkmark$ & $\sqrt{ } \sqrt{ }$ & $x$ \\
\hline Planejamento & $x$ & $\checkmark$ & $x$ & $x$ & $x$ & $x$ \\
\hline Revisões & $x$ & $x$ & $x$ & $x$ & $x$ & $x$ \\
\hline Upload de Arquivos & $\checkmark$ & $\sqrt{ } \sqrt{ }$ & $x$ & $x$ & $x$ & $x$ \\
\hline Autenticação do usuário & $\checkmark$ & $\checkmark$ & $\checkmark$ & $\checkmark$ & $\checkmark$ & $\checkmark$ \\
\hline Critérios atendidos & 10 & 12 & 4 & 4 & 3 & 4 \\
\hline
\end{tabular}

atende parcialmente o requisito

$\checkmark \checkmark$ atende plenamente o requisito

$x \quad$ não atende o requisito

Gobby e ACE foram implementados de tal forma que a edição síncrona é a mais indicada pois para que os autores tenham acesso à última versão do documento, o autor da versão mais recente precisa estar online. Com relação a esta funcionalidade, tanto o Google Docs quanto o Zoho permitem que qualquer autor acesse a última versão do 
documento a qualquer momento. O SynchroEdit é um editor que possui os recursos de chat e percepção de grupo, mas não possui controle sobre os membros que podem acessar o documento, tornando difícil o gerenciamento dos usuários e dos recursos utilizados. Por fim, o EquiText apresenta uma proposta diferenciada pela metodologia de construção do texto. Implicitamente, a construção de um texto acontece através da soma de parágrafos, mas o EquiText torna isso obrigatório. Além disso, este editor implementa poucas funcionalidades.

Os softwares que funcionam como aplicativos locais (Gobby e ACE) oferecem condições para que pequenos documentos possam ser criados de modo colaborativo, pois a carência do controle de versão de arquivos e inclusive de formatação de texto os torna pouco indicados para a produção de documentos mais elaborados. Os demais editores, especialmente Google Docs e Zoho, são mais robustos mas carecem de componentes como fóruns, controle de versões de arquivos e planejamento de tarefas. A partir desta análise, identificou-se que os editores mais completos são Zoho (12 critérios atendidos) e Google Docs (10 critérios atendidos). Analisando a capacidade de reutilização de seus componentes, verificou-se que seria possível desenvolver ferramentas e integrá-las à plataforma Google de maneira eficiente. Desenvolveu-se assim um modelo de editor integrado ao Google Docs denominado DocsCollab. A próxima secção descreve esta ferramenta.

\section{Ferramenta DocsCollab}

A ferramenta DocsCollab foi desenvolvida de forma a integrar funcionalidades importantes para a edição colaborativa mas que não foram encontradas em um única ferramenta. A ferramenta desenvolvida é uma aplicação web para a qual cada autor deve se autenticar fornecendo seu usuário e senha utilizados para a plataforma Google. Uma vez conectado, a ferramenta se integra ao Google Docs e recupera a lista de documentos e co-autores que possuem acesso a cada um dos documentos. A ferramenta possui um fórum cujas mensagens serão vinculadas aos documentos. Os usuários podem selecionar o documento e verificar todas as mensagens vinculadas a ele. Foi implementada também uma árvore de alocação de tarefas que contém as notificações aos usuários para cada documento em edição. As próximas seções detalham o desenvolvimento e as funcionalidades da ferramenta DocsCollab.

O desenvolvimento da ferramenta foi realizado utilizando as bibliotecas GWT (Google Web Toolkit) que permite programar na linguagem JAVA e após a compilação o aplicativo é disponibilizado para o usuário final através de uma página HTML contendo scripts em JavaScript. Como resultado obtém-se uma aplicação em AJAX (Asynchronous Javascript And XML1) que oferece ao usuário uma interface mais ágil, rica e dinâmica, seguindo padrões web atuais. A ferramenta está disponível através de acesso ao seu servidor, onde está instalado seu serviço de banco de dados e servidor de aplicação HTTP. Para carregar estes dados, a aplicação cliente utiliza o mecanismo GWT RPC (Google Web Toolkit Remote Procedure Call).

Os dados solicitados pela máquina cliente ao servidor possuem duas fontes de origem, uma do próprio Google Docs e outra sendo o banco de dados hospedado em nosso servidor. A partir do Google Docs são validados os dados para acesso, obtém-se a lista dos documentos do usuário principal e visualiza-se os demais usuários que possuem acesso a cada um dos documentos. Esses dados são necessários para a exibição da lista dos documentos e para que seja possível vincular os itens gerados pela aplicação, como as mensagens e tarefas, com o identificador único de cada documento. 
O acesso ao sistema é realizado através do usuário e senha cadastrados pelo usuário nos serviços do Google. Foi utilizada a integração disponibilizada pelo Google ${ }^{1}$ para que a aplicação pudesse validar os dados do usuário e permitir a entrada no sistema. Uma vez o usuário esteja conectado, a ferramenta utiliza as API's de integração oferecidas pelo Google e recupera a lista dos documentos (textos, planilhas, apresentações ou outros) do usuário. Esta lista de documentos fica disponível no painel do usuário, ao lado esquerdo da tela (figura 1). Os documentos são exibidos para o usuário utilizando a mesma estrutura de diretórios presentes no Google Docs. $\mathrm{O}$ processo de edição de textos do Google Docs não foi alterado, contudo novas funcionalidades foram implementadas. $\mathrm{Na}$ interface foram criadas duas abas que estão disponíveis para visualização de tarefas e das mensagens do fórum (figura 1).

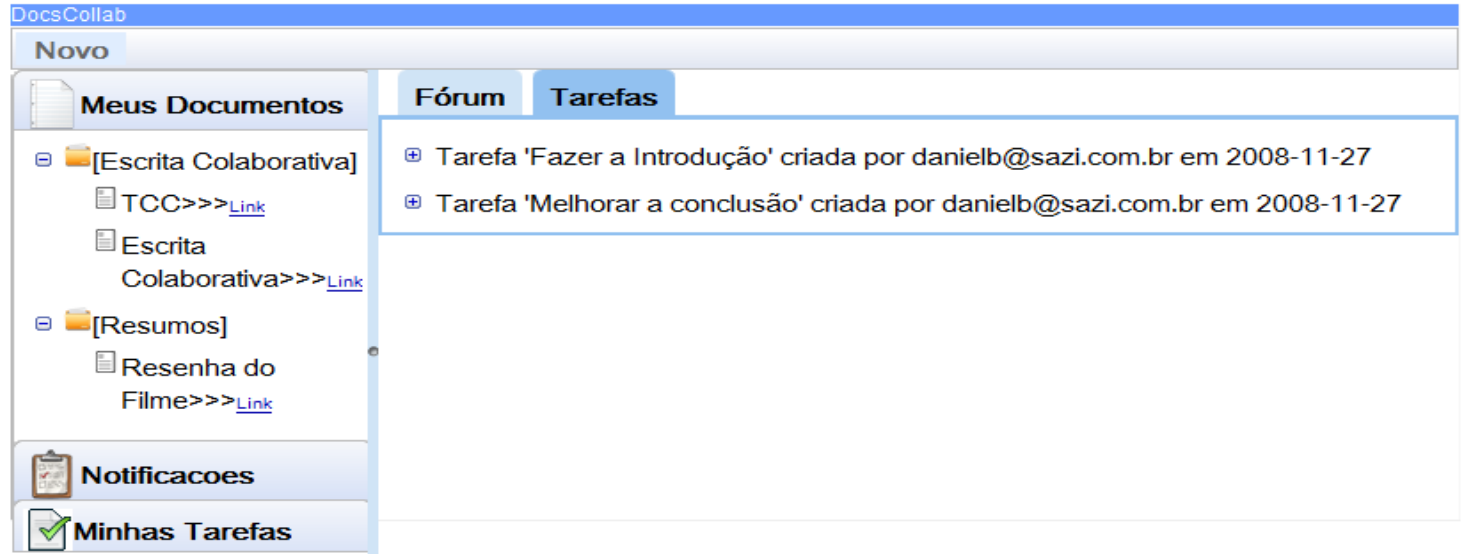

Figura 1. Lista de documentos disponibilizada pela ferramenta

O painel do usuário contém os seus itens, mantendo também a lista de documentos aos quais ele possui acesso, as notificações recebidas e as tarefas que the foram atribuídas. Esses dados são atualizados a cada 2 minutos, uma vez que novos documentos podem ser compartilhados a todos instante. Através do painel principal o usuário tem a possibilidade de acessar os documentos de maneira ágil, bastando clicar no link presente ao lado do nome de cada documento.

\section{Fórum}

As mensagens criadas no fórum devem estar vinculadas a um documento. Para isso, o usuário deve antes de criar a mensagem, selecionar um documento. $\mathrm{O}$ fórum foi desenvolvido também para que as mensagens fiquem organizadas por tópicos de acordo com a organização dos documentos (figura 2).

\begin{tabular}{|c|c|c|}
\hline Novo & & \\
\hline Meus Documentos & $\nabla$ Criar Novo Topico & \\
\hline$\ominus$ [Escrita Colaboratival & |Capitulo 2: Desenvolvimento| & Salvar \\
\hline G TCC & Tarefas & \\
\hline $\begin{array}{c}\text { Escrita } \\
\text { Colaborativa } \\
\oplus \text { [Resumos] }\end{array}$ & $\begin{array}{l}\text { Introdução } \\
\text { Capítulo1: Fundamentação }\end{array}$ & \\
\hline Notificacoes & & \\
\hline Minhas Tarefas & & \\
\hline
\end{tabular}

Figura 2.Interface do fórum

\footnotetext{
1 http://code.google.com/apis/documents/docs/2.0/developers_guide_java.html\#AuthClientLogin

$2 \mathrm{http}: / / \operatorname{code}$. google.com/apis/documents/docs/2.0/developers_guide_java.html
}

V. $9 \mathrm{~N}^{\mathrm{o}}$ 2, dezembro, 2011 
A figura 3 ilustra a tarefa de criação de uma mensagem, no contexto de um documento denominado TCC. O usuário deve digitar um título para a mensagem, o corpo da mensagem e escolher a qual tópico ela estará vinculada.

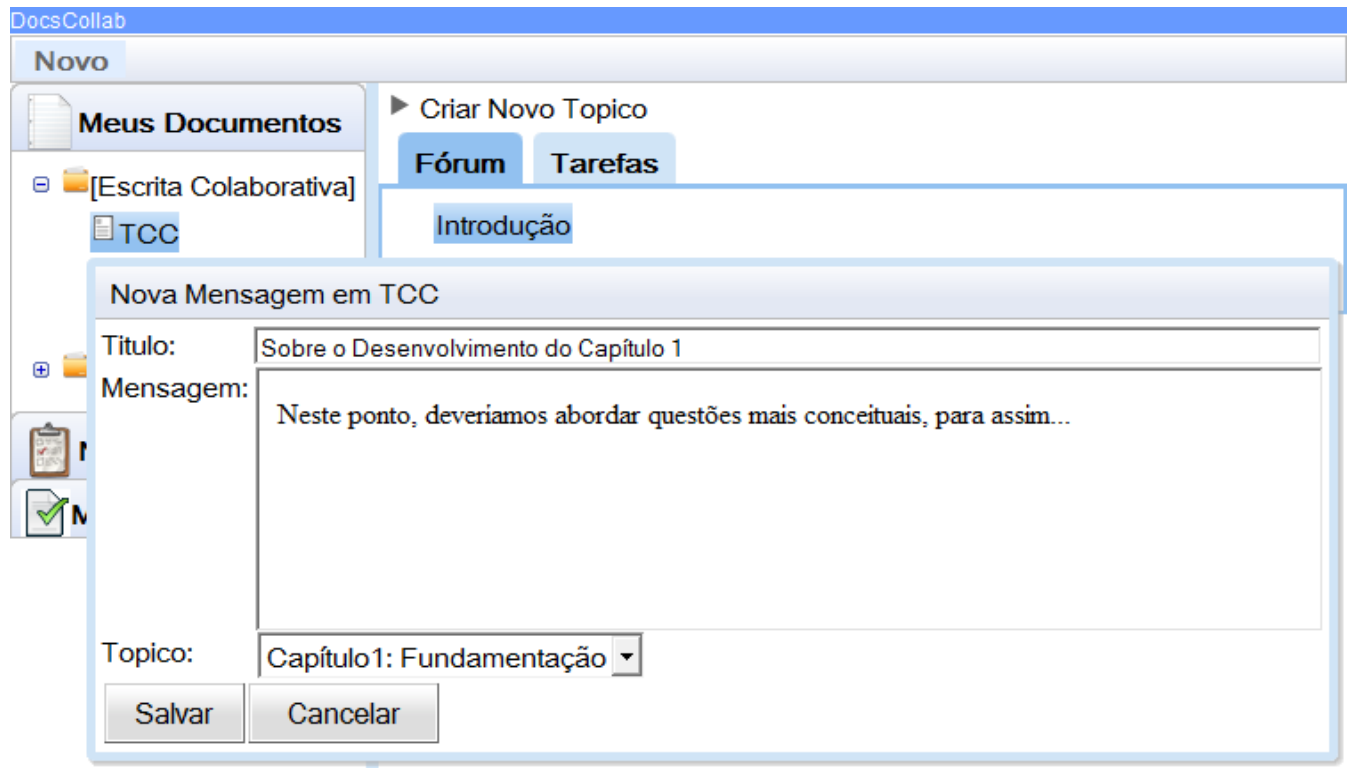

Figura 3. Inclusão de mensagem em um fórum

As mensagens criadas são posteriormente disponibilizadas através da aba 'Fórum' do DocsCollab (figura 4). Elas ficam agrupadas pelos tópicos e ao expandi-los as mensagens são expostas. Esta aba Fórum é atualizada conforme o usuário seleciona o documento desejado no painel do usuário.

\begin{tabular}{|c|c|c|c|}
\hline \multicolumn{4}{|l|}{ JocsCollab } \\
\hline \multicolumn{4}{|l|}{ Novo } \\
\hline Meus Documentos & \multicolumn{3}{|c|}{ Criar Novo Topico } \\
\hline \multirow{2}{*}{$\begin{array}{l}\ominus \text { [Escrita Colaborativa] } \\
\qquad \text { TCC }\end{array}$} & Fórum & \multicolumn{2}{|l|}{ Tarefas } \\
\hline & \multicolumn{3}{|c|}{ Introdução } \\
\hline Escrita & \multicolumn{3}{|c|}{ 曰 Capítulo1: Fundamentação } \\
\hline $\begin{array}{l}\text { Colaborativa } \\
\oplus \text { [Resumos }]\end{array}$ & \multicolumn{3}{|c|}{$\begin{array}{l}\text { Mensagem 'Sobre o desenvolvimento do Capítulo 1' criada por } \\
\text { danielb@sazi.com.br em 2008-11-27 }\end{array}$} \\
\hline Notificacoes & \multicolumn{2}{|r|}{$\begin{array}{l}\text { Nesse ponto, deveríamos abordar questões mais conceituais, } \\
\text { para assim... }\end{array}$} & \\
\hline Minhas Tarefas & \multicolumn{3}{|c|}{ Capitulo 2: Desenvolvimento } \\
\hline
\end{tabular}

Figura 4. Fórum do DocsCollab

\section{Tarefas}

Assim como as mensagens do fórum, as tarefas também são vinculadas aos documentos. O usuário deve selecionar o documento desejado no painel do usuário e em seguida criar a tarefa (figura 5). Na criação da tarefa, além do título, da descrição da tarefa e da data de entrega, deve ser selecionado um ou mais usuários responsáveis pela conclusão desta tarefa. Uma vez criada, a tarefa aparece no painel dos seus autores. Ela só estará finalizada quando todos os autores tiverem assinalado a tarefa como finalizada. 


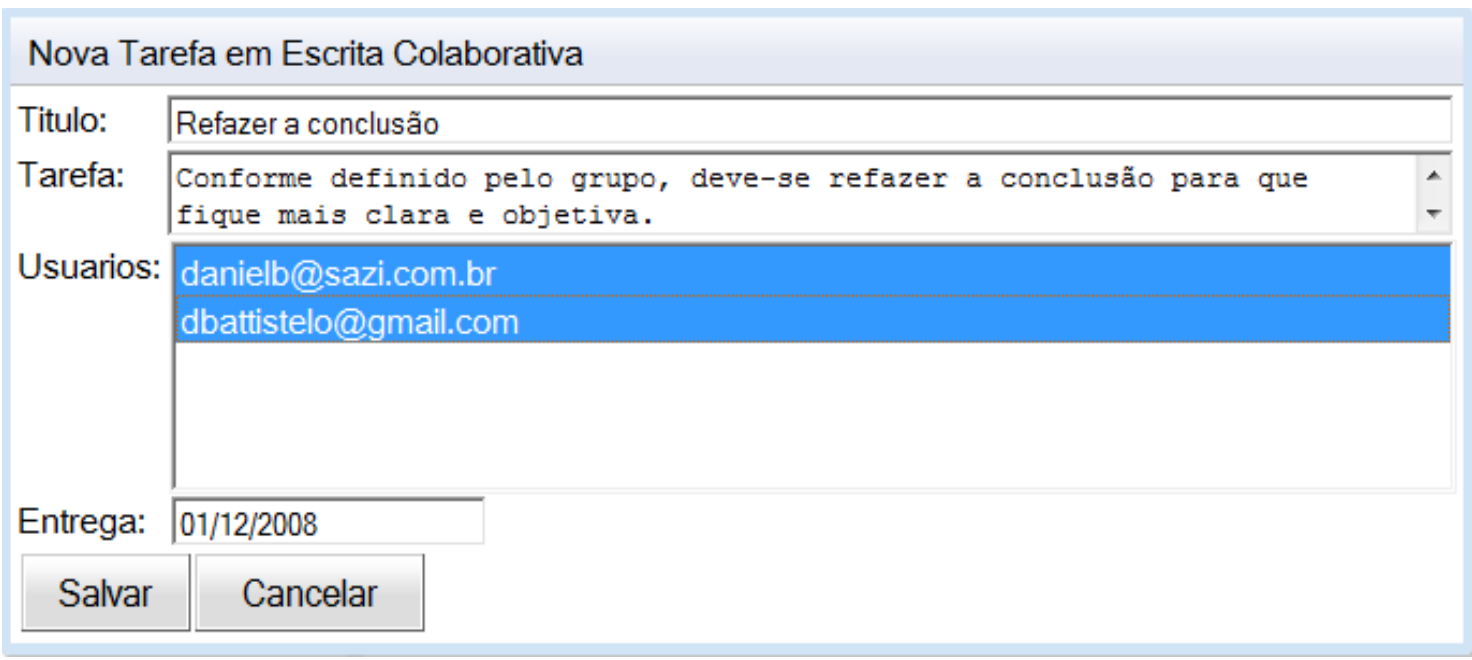

Figura 5. Interface de especificação de tarefas

A figura 6 apresenta a interface do usuário. Nela pode-se visualizar as tarefas alocadas ao usuário e a árvore de alocação.

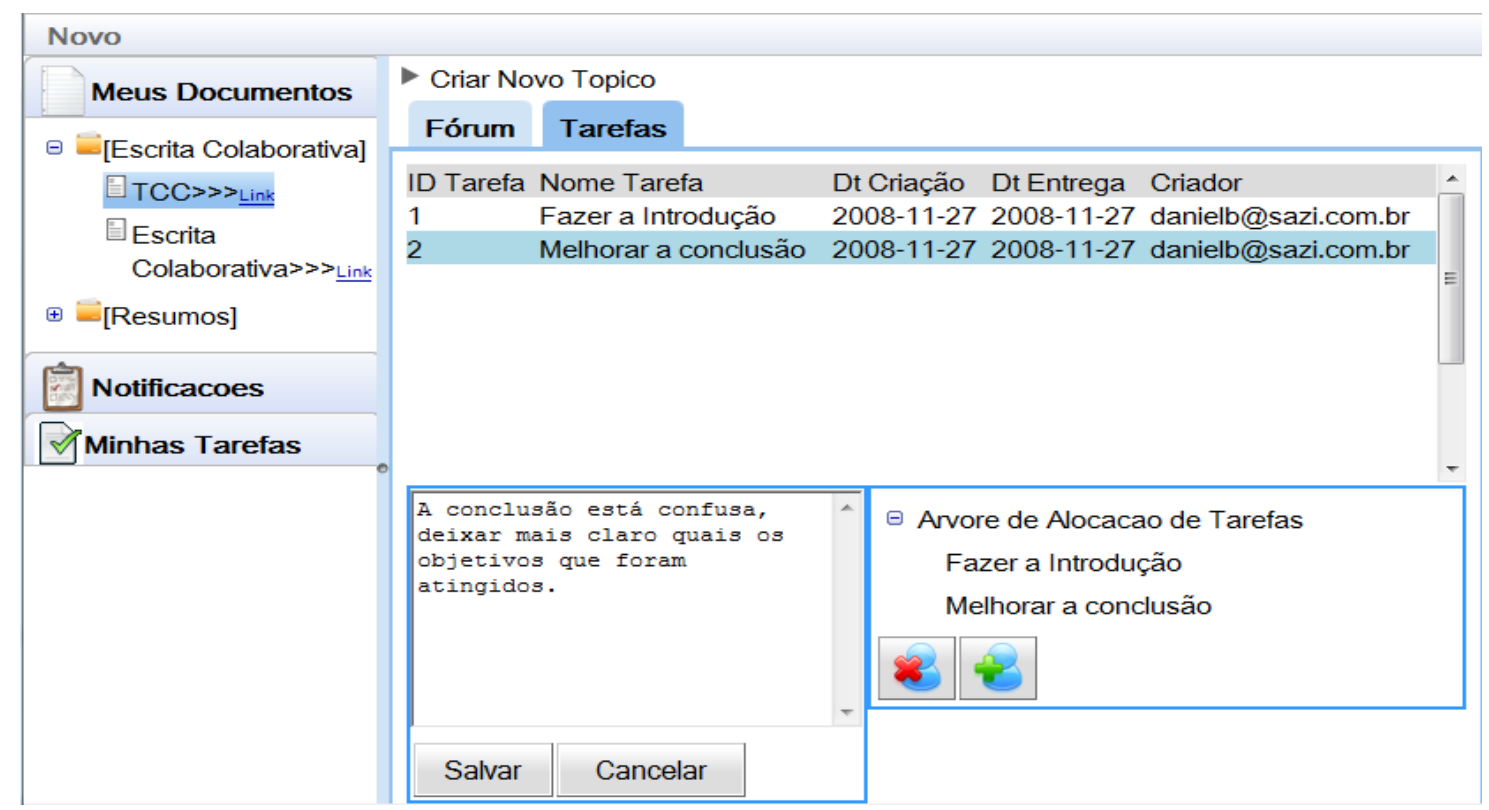

Figura 6. Visualização das Tarefas no DocsCollab

$\mathrm{Na}$ parte superior da interface há uma lista com as tarefas ativas relacionadas ao documento selecionado (TCC). Ao selecionar-se uma das tarefas, o painel inferior é atualizado contendo a descrição da tarefa e a árvore de alocação de tarefas. Através do componente árvore de alocação de tarefas os autores podem ser excluídos ou adicionados. Os testes da ferramenta demonstraram que as novas funcionalidades são úteis e complementam a aplicação Google Docs. A documentação detalhada desta ferramenta está disponível em (Battistelo, 2010).

\section{Considerações Finais}

A edição colaborativa é uma tarefa rica que pode ser desenvolvida com fins educacionais em disciplinas e faixas etárias variadas. Através deste estudo pretendeu-se 
estudar e testar editores colaborativos, identificando funcionalidades presentes e relevantes para o seu uso em contextos de ensino-aprendizagem. De fato, percebeu-se ao longo deste trabalho que a escrita colaborativa é uma tendência muito forte e bem recebida tanto por professores quanto por estudantes pois estamos cada vez mais conectados uns aos outros e assim nos habituados a compartilhar informações. Seja através de redes sociais, de sistemas de comunicação ou blogs, a troca de informações em formato textual e sua disponibilização a um grupo ou comunidade já faz parte da vida das crianças e adolescentes. Cabe aos educadores explorar tais habilidades para fins educacionais valendo-se para isso de ferramentas de escrita colaborativa, por exemplo. Embora diversos editores já estejam disponíveis, novas ferramentas estão constantemente sendo desenvolvidas, oferecendo novas funcionalidades e possibilitando aos usuários atividades de colaboração cada vez mais produtivos e eficientes, seja para a produção de documentos executivos ou em contextos educacionais.

Em nossa análise, identificamos que as ferramentas Zoho Writer e Google Docs se destacam pela variedade de recursos que podem ser explorados na escrita colaborativa. Outras ferramentas como Gobby, ACE e Synchro Edit permitem a criação e edição de documentos com recursos de colaboração mais simples. A partir deste estudo foi desenvolvido um conjunto de funcionalidades para compor a ferramenta DocsCollab e assim agregar recursos ao Google Docs. Desejou-se desenvolver uma ferramenta integrada que somasse recursos de edição síncrona e um ambiente de colaboração mais completo, fazendo com que alguns os recursos de comunicação, cooperação e coordenação fossem vinculados a cada documento compartilhado.

Para trabalhos futuros, outros recursos poderiam ser implementados, como uma ferramenta para o planejamento das atividades e agenda para o grupo. Além disso, pretende-se generalizar a biblioteca de funcionalidades para que ela seja disponibilizada para outros editores colaborativos. Para finalizar, pretende-se realizar testes da ferramenta em situações de aprendizagem colaborativa.

\section{Referências}

ACE. Editor Colaborativo. Disponível em http $/ /$ sourceforge.net/projects/ace

ADKINS, J., REINIG,Q., KRUSE, J. (1999). GSS Collaboration in Document Development: Using GroupWriter to Improve the Process, University of Arizona.

BATTISTELO, D. (2010). DocsCollab - Ferramenta para apoio à Edição Colaborativa. Universidade de Caxias do Sul, Centro de Computação e Tecnologia da Informação.

CALVO, R. A., O'ROURKE, S.T., JONES, J., YACE, K., REIMANN, P. (2011). Collaborative Writing Support Tools on the Cloud," IEEE Transactions on Learning Technologies, pp. 88-97, January-March.

DUIN, A. (1990). Terms and tools: A theory and research-based approach to collaborative writing, Business Communication Quarterly, Vol. 53, No. 2, pp 45-50.

EQUITEXT. Editor Colaborativo. Disponível em http $/ /$ equitext.pgie.ufrgs.br

FISH, R.S., KRAUT, R., LELAND, M. D. P. (1988). Quilt: a collaborative tool for cooperative writing, University Of Washington.

FUKS, H., GEROSA, M.A., LUCENA, C.J.P. (2001). Sobre o Desenvolvimento e Aplicação de Cursos Totalmente a Distância na Internet. Revista Brasileira de Informática na Educação, n.9, setembro, Sociedade Brasileira de Computação, pp. 6175.

GOBBY. Editor Colaborativo. Disponível em http://gobby.0x539.de 
GOOGLE Docs. Editor Colaborativo. Disponível em http://docs.google.com

GUTWIN, C., GREENBERG, S. (2001). A Descriptive Framework of Workspace Awareness for Real-Time Groupware. Computer Supported Cooperative Work, Kluwer Academic Press.

NEUWIRTH, C.M., KAUFER, D.S., CHANDHOK, R., MORRIS, J. (1994). Computer Support for Distributed Collaborative Writing: Defining Parameters of Interaction,.

RESNICK, L. B. (1991). Shared Cognition: thinking as social practice. In L. B. Resnick \& J. M. Levine \& S. D. Teasley (Eds.), Perspectives on Socially Shared Cognition. Arlington VA: American Psychological Association. p.1-20.

SALOMON, G., \& PERKINS, D. N. (1998). Individual and Social Aspects of Learning. Review of Research in Education, 23, p.1-24.

SYNCHROEDIT. Editor Colaborativo. Disponível em http://www. synchroedit.com

TRAN, M.H.,YANG,Y., RAIKUNDALIA, K.G. (2006). Extended Radar View and Modification Director: Awareness Modification for Synchronous Collaborative Authoring.

VYGOTSKY, L. S. (1962). Thought and Language. E. Hanfmann and G. Vakar, Trans. Cambridge, MA: MIT Press.

VYGOTSKY, L. S. (1978). Mind in Society: the development of higher psychological processes. M. Cole, V. John-Steiner, S. Scribner and E. Souberman, Trans. Cambridge MA: Harvard University Press.

WENG, C., GENAARI, J. (2004). Asynchronous Collaborative Writing through Annotations. In Proceedings of the ACM Conference on Computer Supported Cooperative Work, Chicago, Illinois, USA, pp $578-581$.

WOO, M., CHU, S., HO, A., LI, X. (2011). Using a Wiki to Scaffold Primary-School Students' Collaborative Writing. Educational Technology \& Society, 14 (1), 43-54.

YANG, Y., SUN, C., ZHANG, Y., JIA, X. (2000). Realtime Cooperative Editing on the Internet, IEEE Internet Computing, 4(1):18-25.

ZOHO Editor. Editor Colaborativo. Disponível em http://writer.zoho.com 